Bull. Korean Math. Soc. 51 (2014), No. 4, pp. 1015-1022

http://dx.doi.org/10.4134/BKMS.2014.51.4.1015

\title{
ON SOME OPEN PROBLEMS OF MAHMOUDI AND RENSHAW
}

\author{
Husheng Qiao and Chongqing Wei
}

\begin{abstract}
This paper continues the investigation of covers of cyclic acts over monoids. Special attention is paid to (P)-covers and strongly flat covers of cyclic acts. In 2008 Mahmoudi and Renshaw posed some open problems and we gave some examples on them in 2012. In this paper, we obtained some further results on these problems and hence gave some deeper answers to them.
\end{abstract}

\section{Introduction}

Throughout this paper, $S$ always stands for a monoid, and $\mathbb{N}$ the set of nonnegative integers.

The substantial works on covers of acts over monoids are referred to the following articles: Isbell [4], Fountain [2] and Kilp [6]. These authors mainly consider projective covers. Further investigations about this fields have lain dormant until the appearance of the article Mojgan Mahmoudi and James Renshaw [9]. Mahmoudi and Renshaw define a strongly flat cover ((P)-cover) and give a necessary and sufficient condition for the existence of such covers and for a monoid to have the property that all its cyclic right acts have a strongly flat cover (resp. (P)-cover). They also give a new necessary and sufficient condition for a cyclic act to have a projective cover and provide a new proof of one of Isbells classic results concerning projective covers. At the end of this article, the authors posed some open questions for consideration, the following are two of them:

1. Is there a monoid $S$ and a cyclic $S$-act $A$ which does not have a (P)-cover? (The open problem 1 in [9].)

2. Are strongly flat covers unique? (The open problem 5 in [9].)

Later in [5], the authors characterized monoids over which every right $S$-act has a strongly flat (condition (P)) cover. They also gave a new characterization for perfect monoids as monoids over which every strongly flat right $S$-act has a projective cover. But the above problems still remains open. Especially in [1],

Received August 1, 2013; Revised January 29, 2014.

2010 Mathematics Subject Classification. 20M30.

Key words and phrases. (P)-cover, strongly flat cover, cyclic act. 
Ershad and Khosravi pointed out that they do not know whether the strongly flat cover of cyclic acts are unique, hence they gave numerous classes of monoids over which strongly flat covers of cyclic acts are unique.

In [10] we have given some examples to show that the answer of question 1 is affirmative, the answer of question 2 is negative. But for $(\mathrm{P})$-cover of cyclic acts, we only proved that there exists a monoid such that the special cyclic act, 1-element $S$-act $\{\Theta\}$ has no $(\mathrm{P})$-cover, we did not find a monoid and a general cyclic act $A$ but not 1-element $S$-act $\{\Theta\}$ over which $A$ has no (P)-cover. And if a cyclic act $A$ has $(\mathrm{P})$-covers, we also do not know how many covers it can have. Also in [10], for question 2, we gave an example of finite semigroup to show that strongly flat covers of cyclic acts are not unique, but we do not know the number of strongly flat covers of a cyclic act.

In this article, we obtain some further results on these problems and hence gave some new answers to them. We prove that for any cardinal number $\alpha$, there always exists a cyclic $S$-act $A$ such that number of $(\mathrm{P})$-covers of $A$ is $\alpha$.

We refer the reader to [3] for basic definitions and results on semigroup theory, and to [8] for various flatness properties of acts over monoids. Now let us recall results and definitions we shall use below.

In [12], the acts now called strongly flat were introduced: a right $S$-act $A_{S}$ is strongly flat if the functor $A_{S} \bigotimes$ - preserves pullbacks and equalizers. In the same article, strongly flat acts were characterized as those acts satisfy two interpolation conditions, later labelled condition $(\mathrm{P})$ and condition $(\mathrm{E})$ :

(P) $\quad\left(\forall a, a^{\prime} \in A\right)(\forall s, t \in S)$

$$
\left(a s=a^{\prime} t \Rightarrow\left(\exists a^{\prime \prime} \in A\right)(\exists u, v \in S)\left(a=a^{\prime \prime} u \wedge a^{\prime}=a^{\prime \prime} v \wedge u s=v t\right)\right)
$$

(E) $\quad(\forall a \in A)\left(\forall s, s^{\prime} \in S\right)$

$$
\left(a s=a s^{\prime} \Rightarrow\left(\exists a^{\prime} \in A\right)(\exists u \in S)\left(a=a^{\prime} u \wedge u s=u s^{\prime}\right)\right) .
$$

Definition 1.1 ([9, Definition 2.1]). Let $S$ be a monoid and A an $S$-act. We shall say that an $S$-act $C$ together with an $S$-epimorphism $f: C \rightarrow A$ is a cover of A if there has no proper subact $B$ of $C$ such that $f \mid B$ is onto. We shall usually refer to $C$ as the cover. Whenever $C$ is a free (projective, strongly flat) $S$-act, then $C$ is called a free (projective, strongly flat) cover.

Definition 1.2 ([9, Definition 2.2]). Let $S$ be a monoid and $f: C \rightarrow A$ be an $S$-epimorphism. We call $f$ coessential if for each $S$-act $B$ and each $S$-map $g: B \rightarrow C$, if $f g$ is an epimorphism, then $g$ is an epimorphism.

It is easy to see that $f: C \rightarrow A$ is a cover of $A$ if and only if it is a coessential epimorphism.

\section{Main results}

Let $X$ be a nonempty set, and let $X^{+}$denote the free semigroup generated by $X$. If we adjoin an identity 1 to $X^{+}$we obtain the free monoid on $X$ and we denote it by $X^{*}$. For each $w$ in $X^{+}$, by [3] the content $C(w)$ is defined as the 
(necessarily finite) set of elements of $X$ appearing in $w$. Let $R$ be a submonoid of a free monoid $X^{*}$. We define the content $C(R)$ of $R$ as

$$
C(R)=\bigcup_{w \in R} C(w) .
$$

If $R=\{1\}$, we denote $C(R)=\emptyset$ and hence $R=\{1\}$ if and only if $|C(R)|=0$.

A monoid $S$ is said to be right reversible if for any $p, q \in S$, there exist $u, v \in S$ such that $u p=v q$.

Lemma 2.1 ([10, Lemma 2.2]). Let $X$ be a non-empty set and let $R$ be a subsemigroup of $X^{+}$. Then $R$ is right reversible if and only if $|C(R)|=1$.

Lemma $2.2([9$, Theorem 4.2]). Let $S$ be a monoid. Then the cyclic $S$-act $S / \rho$ has a $(\mathrm{P})$-cover if and only if $[1]_{\rho}$ contains a right reversible submonoid $R$ such that for all $u \in[1]_{\rho}, u S \cap R \neq \emptyset$.

Theorem 2.3. Let $X$ be a set with more than 2 elements and $S=X^{*}$, the free monoid generated by $X$. Suppose $\rho$ is a right congruence of $S$. Then

(1) The cyclic right $S$-act $S / \rho$ has no $(\mathrm{P})$-cover if and only if $\left|C\left([1]_{\rho}\right)\right|>1$;

(2) The cyclic right $S$-act $S / \rho$ has a $(\mathrm{P})$-cover if and only if $\left|C\left([1]_{\rho}\right)\right|=0$ or $\left|C\left([1]_{\rho}\right)\right|=1$.

Proof. (1) Necessity: Let $\left|C\left([1]_{\rho}\right)\right|=n$, where $n$ is a nonnegative integer. If $n=0$, then $[1]_{\rho}=\{1\}$, its only right reversible submonoid is $\{1\}$ and by Lemma 2.2 it is clear that the cyclic right $S$-act $S / \rho$ has a $(\mathrm{P})$-cover, which is a contradiction. If $n=1$, we denote $C\left([1]_{\rho}\right)=\{x\}$, then every element of $[1]_{\rho}$ is the power of $x$. For any $s, t \in[1]_{\rho}$, there exist positive integers $k, l$ such that $s=x^{k}, t=x^{l}$. Hence $s t=t s$ implies [1] $]_{\rho}$ is right reversible. And for all $u \in[1]_{\rho}, u S \cap[1]_{\rho} \neq \emptyset$. So by Lemma 2.2 the cyclic right $S$-act $S / \rho$ has a (P)-cover, which is also a contradiction. Now the result follows.

Sufficiency: Assume the cyclic right $S$-act $S / \rho$ has a (P)-cover, then by Lemma $2.2[1]_{\rho}$ contains a right reversible submonoid $R$ such that for all $u \in$ $[1]_{\rho}, u S \cap R \neq \emptyset$. By Lemma $2.1|C(R)|=1$, but $\left|C\left([1]_{\rho}\right)\right|>1$ and $S$ is a free monoid, which is a contradiction.

(2) Since for every cyclic right $S$-act $S / \rho$, either it has a (P)-cover or it has not one, then it is proved similar to (1).

Remark 2.4. Theorem 2.3 gives an answer to the open problem 1 in [9]: Is there a monoid $S$ and a cyclic $S$-act $A$ which does not have a (P)-cover? By Theorem 2.3 we only need to choose an arbitrary free monoid $\mathrm{S}$ and its arbitrary right congruence $\rho$ satisfying $\left|C\left([1]_{\rho}\right)\right|>1$, then the cyclic right $S$-act $S / \rho$ has no (P)-cover.

Corollary 2.5 ([10, Example 2.4]). Let $X$ be a set with more than 3 elements and $S=X^{*}$, the free monoid generated by $X$. Then the (cyclic) 1-element $S$-act $\{\Theta\}$ has no $(\mathrm{P})$-cover. 
The following Example 2.6 gave a proper congruence $\rho$ such that cyclic $S$-act $S / \rho$ has no $(\mathrm{P})$-cover.

Example 2.6. Let $S=\langle x, y, z \mid x z=z x=y z=z y=z\rangle \cup\{1\}$. Define an equivalence relation $\rho$ on $S$ by

$$
s \rho t \Longleftrightarrow s, t \in\langle z\rangle \text { or } s, t \in\langle x, y\rangle \cup\{1\} .
$$

It is easy to verify that $\rho$ is a right congruence on $S$. Then $[1]_{\rho}=\langle x, y\rangle \cup\{1\}$ and the cyclic right $S$-act $S / \rho$ has no $(\mathrm{P})$-cover.

Proof. By the definition of $\rho$, it is a proper right congruence of $S$. If $R$ is a right reversible submonoid of $[1]_{\rho}$, Lemma 2.1 implies that $|C(R)|=1$, we have the following two cases to consider:

Case 1: $C(R)=\{x\}$. Then $y \in[1]_{\rho}$, but $y S \cap R=\emptyset$, by Lemma $2.2 S / \rho$ has no $(\mathrm{P})$-cover.

Case 2: $C(R)=\{y\}$. Then $x \in[1]_{\rho}$, but $x S \cap R=\emptyset$, by Lemma $2.2 S / \rho$ has no $(\mathrm{P})$-cover.

A monoid $S$ is said to be weakly left collapsible if for any $s, t, z \in S, s z=t z$ implies that there exists $u \in S$ such that $u s=u t$.

Lemma 2.7 ([10, Theorem 4.3]). Let $S$ be a monoid. Then the cyclic $S$-act $S / \rho$ has a weakly pullback flat cover if and only if $[1]_{\rho}$ contains a right reversible and weakly left collapsible submonoid $R$ such that for all $u \in[1]_{\rho}, u S \cap R \neq \emptyset$.

When a monoid $S$ is a free monoid, every right $S$-act $A$ satisfies condition (P) if and only if $A$ is weakly pullback flat. Hence by Theorem 2.3 we have the following.

Proposition 2.8. There is a monoid $S$ and a cyclic $S$-act $A$ which does not have a weakly pullback flat cover.

Lemma 2.9 ([11, Lemma 2.1]). Let $P \subseteq S$ be a right reversible submonoid and let $\rho$ be the relation on $S$ defined by

$$
s \rho s^{\prime} \Leftrightarrow(\exists p, q \in P)\left(p s=q s^{\prime}\right) .
$$

Then

(1) $\rho$ is a right congruence.

(2) $S / \rho$ satisfies condition $(\mathrm{P})$.

Lemma 2.10 ([9, Theorem 2.7]). Let $S$ be a monoid and $S / \rho$ a cyclic $S$-act. The map $f: S / \sigma \rightarrow S / \rho$ given by $s \sigma \mapsto s \rho$ is a coessential epimorphism if and only if

$$
\sigma \subseteq \rho \text { and for all } u \in[1]_{\rho}, u S \cap[1]_{\sigma} \neq \emptyset .
$$

Given any cardinal number $\alpha$, the following Theorem 2.11 shows that there exist a monoid $S$ and a cyclic $S$-act $A$ whose number of (P)-covers is $\alpha$.

Theorem 2.11. Given any cardinal number $\alpha$, there always exists a cyclic right $S$-act $A$ such that the number of $(\mathrm{P})$-covers of $A$ is $\alpha$. 
Proof. Assume $X$ be a nonempty set and its cardinal number is $\alpha$. Denote $X=\left\{x_{i} \mid i \in I\right\}$ and choose an element $y \notin X$. Let $S_{1}=\left\langle x_{i}\right| x_{i} x_{j}=x_{j}^{2}, i, j \in$ $I\rangle, S_{2}=y^{+}$be a free semigroup generated by $y$, and $S=S_{1} \cup S_{2} \cup\{1\}$. It is clear $S_{1} \cap S_{2}=\phi$. We define the multiplication on $S$ as follows:

$u v=v u=v$ for any $u \in S_{1}$ and $v \in S_{2} ; \quad 1 w=w 1=w$ for any $w \in S$.

Define a relation $\rho$ on $S$ by

$$
s \rho t \Longleftrightarrow s, t \in S_{1} \cup\{1\} \text { or } s, t \in S_{2} \text {. }
$$

It is clear that $\rho$ is an equivalent relation on $S$, and by definition of monoid $S$, it is easy to verify that $\rho$ is a right congruence on $S$ and $[1]_{\rho}=S_{1} \cup\{1\}$.

Denote $R_{i}=x_{i}^{+} \cup\{1\}\left(x_{i} \in X\right)$. By Lemma 2.1 every $R_{i}$ is a right reversible submonoid of $S$. By Lemma 2.9 we can define a right congruence $\sigma_{i}$ on $S$ by

$$
s \sigma_{i} t \Longleftrightarrow\left(\exists p, q \in R_{i}\right)(p s=q t) .
$$

Now by Lemma 2.9 every cyclic right $S$-act $S / \sigma_{i}$ satisfies condition $(\mathrm{P})$. And if $i \neq j$, then $\sigma_{i} \neq \sigma_{j}$, since $\left(x_{i}, 1\right) \in \sigma_{i}$ but $\left(x_{i}, 1\right) \notin \sigma_{j}$, and $\left(x_{j}, 1\right) \in \sigma_{j}$ but $\left(x_{j}, 1\right) \notin \sigma_{i}$.

By definition of $S$, for every $u \in[1]_{\rho}, u S \cap[1]_{\sigma_{i}} \neq \emptyset(i \in I)$. We now show that $\sigma_{i} \subseteq \rho$. For any $s, t \in S$ and $s \sigma_{i} t$, there exist $p, q \in R_{i}$ such that $p s=q t$. We assert that $s \rho t$. Otherwise if $s \in S_{1} \cup\{1\}$ and $t \in S_{2}$, then by definition of $S, p s \in S_{1}$ and $q t \in S_{2}$, which is a contradiction. Another case is similar.

Hence by Lemma $2.10 S / \sigma_{i}$ are all $(\mathrm{P})$-covers of $S / \rho$ and the number of (P)-covers of $S / \rho$ is $\alpha$.

A monoid $S$ is said to be left collapsible if for any $p, q \in S$, there exists $u \in S$ such that $u p=u q$.

Lemma 2.12 ([7, Lemma 2.1]). Let $P \subseteq S$ be a left collapsible submonoid and let $\rho$ be the relation on $S$ defined by

$$
s \rho s^{\prime} \Leftrightarrow(\exists p, q \in P)\left(p s=q s^{\prime}\right) .
$$

Then

(1) $\rho$ is a right congruence.

(2) $S / \rho$ is strongly flat.

A monoid $S$ is said to be aperiodic if for any $x \in S$, there exists a positive integer $n$ such that $x^{n}=x^{n+1}$.

Theorem 2.13. Given any cardinal number $\alpha$, there always exists a cyclic right $S$-act $A$ such that the number of strongly flat covers of $A$ is $\alpha$.

Proof. Assume $X$ be a nonempty set and its cardinal number is $\alpha$. Denote $X=\left\{x_{i} \mid i \in I\right\}$ and choose an element $y \notin X$. Let $S_{1}=\left\langle x_{i}\right| x_{i} x_{j}=$ $\left.x_{j}^{2}, i, j \in I\right\rangle$ and $S_{1}$ is aperiodic, $S_{2}=y^{+}$is a free semigroup generated by $y$, 
and $S=S_{1} \cup S_{2} \cup\{1\}$, it is clear $S_{1} \cap S_{2}=\phi$. We define the multiplication on $S$ as follows:

$$
u v=v u=v \text { for any } u \in S_{1} \text { and } v \in S_{2} ; \quad 1 w=w 1=w \text { for any } w \in S .
$$

Define a relation $\rho$ on $S$ by

$$
s \rho t \Longleftrightarrow s, t \in S_{1} \cup\{1\} \text { or } s, t \in S_{2} .
$$

It is clear that $\rho$ is an equivalent relation on $S$, and by definition of monoid $S$, it is easy to verify that $\rho$ is a right congruence on $S$ and $[1]_{\rho}=S_{1} \cup\{1\}$.

Denote $R_{i}=x_{i}^{+} \cup\{1\}\left(x_{i} \in X\right)$. By definition of $S_{1}$, it is obvious that $R_{i}$ is a left collapsible submonoid of $S$. By Lemma 2.12, we define a right congruence $\sigma_{i}$ on $S$ by

$$
s \sigma_{i} t \Longleftrightarrow\left(\exists p, q \in R_{i}\right)(p s=q t) .
$$

By Lemma 2.12 every cyclic right $S$-act $S / \sigma_{i}$ is strongly flat. And if $i \neq j$, then $\sigma_{i} \neq \sigma_{j}$, since $\left(x_{i}, 1\right) \in \sigma_{i}$ but $\left(x_{i}, 1\right) \notin \sigma_{j}$, and $\left(x_{j}, 1\right) \in \sigma_{j}$ but $\left(x_{j}, 1\right) \notin \sigma_{i}$.

By definition of $S$, for every $u \in[1]_{\rho}, u S \cap[1]_{\sigma_{i}} \neq \emptyset(i \in I)$. We now show that $\sigma_{i} \subseteq \rho$. For any $s, t \in S$ and $s \sigma_{i} t$, then there exist $p, q \in R_{i}$ such that $p s=q t$. We assert that sot. Otherwise if $s \in S_{1} \cup\{1\}$ and $t \in S_{2}$, then by definition of $S, p s \in S_{1}$ and $q t \in S_{2}$, which is a contradiction. Another case is similar.

Hence by Lemma $2.10 S / \sigma_{i}$ are all strongly flat covers of $S / \rho$.

Remark 2.14. Theorem 2.13 gives an answer to the open problem 5 in [9]: Are strongly flat covers unique? That is, there exist a monoid $\mathrm{S}$ and a right congruence on $S$ such that the covers of cyclic right $S$-act $S / \rho$ is not unique. More importantly, Theorem 2.11 and Theorem 2.13 show that there always exist a monoid and a cyclic act $A$ such that $A$ has as many strongly flat covers or $(\mathrm{P})$-covers as we want. Hence the covers of $S$-acts are absolutely different from the covers of modules over a ring $R$. Because loss of uniqueness, perhaps it is significative to find the definition of cover of any $S$-act $A$ such that its cover is unique.

Lemma 2.15 ([9, Theorem 3.2]). Let $S$ be a monoid. Then the cyclic $S$-act $S / \rho$ has a strongly flat cover if and only if $[1]_{\rho}$ contains a left collapsible submonoid $R$ such that for all $u \in[1]_{\rho}, u S \cap R \neq \emptyset$.

Theorem 2.16. Let $X$ be a set with more than 2 elements and $S=X^{*}$, the free monoid generated by $X$. Suppose $\rho$ is a right congruence of $S$. Then

(1) The cyclic right $S$-act $S / \rho$ has only strongly flat cover $S$ if and only if $\left|C\left([1]_{\rho}\right)\right|=0$;

(2) The cyclic right $S$-act $S / \rho$ has no strongly flat cover if and only if $\left|C\left([1]_{\rho}\right)\right| \geq 1$.

Proof. (1) Necessity: Since the cyclic right $S$-act $S / \rho$ has a strongly flat cover, by Theorem 2.3 we only need to prove that if $\left|C\left([1]_{\rho}\right)\right|=1$, then $S / \rho$ also has no strongly flat cover. Denote $[1]_{\rho}=\{x\}$, since $S$ is free, the only left collapsible 
submonoid of $[1]_{\rho}$ is $\{1\}$. Hence by Lemma $2.15 S / \rho$ also has no strongly flat cover.

Sufficiency: Assume the cyclic right $S$-act $S / \rho$ has a strongly flat cover $S / \sigma$. For any $s, t \in S$, if $(s, t) \in \sigma$, then there exists $u \in S$ such that $u s=u t$ and $u \rho 1$. Hence $s=t$ and $\sigma=1$.

(2) By the proof of (1) it is clear.

A monoid $S$ is said to be an inverse semigroup if $S$ is regular, and its idempotents commute.

In [9] the author also posed the open problem: Do infinite inverse semigroups have the property that all cyclic S-acts have a (P)-cover? (The open problem 2 in [9].) In the following we will consider this problem.

Proposition 2.17. Let $S$ be an infinite inverse monoid and $\rho$ a right congruence of $S$. If for all $u \in[1]_{\rho}, u u^{-1} \in[1]_{\rho}$, then cyclic right $S$-act $S / \rho$ has a (P)-cover.

Proof. Suppose $S$ is an infinite inverse monoid and $\rho$ is a right congruence of $S$. For $u \in[1]_{\rho}$, by assumption $u u^{-1} \in[1]_{\rho}$. Let $R=\left\{u u^{-1} \mid u \in[1]_{\rho}\right\}$. Since $S$ is an inverse monoid, $R$ is a right reversible submonoid of $[1]_{\rho}$. It is clear that for all $u \in S, u u^{-1} \in R$. By Lemma 2.2 the result follows.

Lemma 2.18 ([9, Theorem 4.2]). The 1-element $S$-act $\{\Theta\}$ has a $(\mathrm{P})$-cover if and only if there exists a right reversible submonoid $R$ of $S$ such that for all $u \in S$, there exists $s \in S$ with us $\in R$.

Corollary 2.19. Let $S$ be an infinite inverse monoid. Then the 1-element $S$-act $\{\Theta\}$ always has a $(\mathrm{P})$-cover.

Proof. Since $[1]_{\rho}=S$ and by Proposition 2.17 and Lemma 2.18 we are done.

The submonoid $T$ of $S$ is said to be left unitary if and only if whenever $t, t s \in T$ then $s \in T$. $T$ is said to be right unitary if and only if whenever $t, s t \in T$ then $s \in T$.

Lemma 2.20 ([4, Section 1.3]). Let $T$ be a submonoid of a monoid $S$. Then $T=[1]_{\rho}$ for some right congruence $\rho$ if and only if $T$ is a left unitary submonoid of $S$.

Hence we have the following result.

Theorem 2.21. Let $S$ be an infinite inverse monoid. If all left unitary submonoids of $S$ are right unitary, then all cyclic S-acts have a $(\mathrm{P})$-cover.

Proof. Suppose $S$ is an infinite inverse monoid and $\rho$ is a right congruence of $S$. By Lemma $2.20[1]_{\rho}$ is a left unitary submonoid of $S$, by assumption $[1]_{\rho}$ is right unitary. For every $u \in[1]_{\rho}, u \rho 1$ implies $u u^{-1} u \rho u^{-1} u$, it means $u^{-1} u \rho 1$. Since $[1]_{\rho}$ is a right unitary submonoid of $S, u^{-1} \in[1]_{\rho}$. Now let $R=\left\{u u^{-1} \mid u \in[1]_{\rho}\right\}$ and it is clear $1 \in R$. Since for every $u \in S, u u^{-1}$ is an idempotent element and 
$S$ is an inverse monoid, $R$ is a right reversible submonoid of $[1]_{\rho}$. By Lemma 2.2 the result follows.

Acknowledgements. We would like to appreciate the anonymous referee for many invaluable comments and suggestions, and we also thank Mojgan Mahmoudi and James Renshaw for posing the questions for consideration. This research was supported by NSFC(No.10901129, No.11101197, No.11001222) and Foundation for Young Innovative Scientists in Gansu Province.

\section{References}

[1] M. Ershad and R. Khosravi, On the uniqueness of strongly flat covers of cyclic acts, Turkish J. Math. 35 (2011), no. 3, 437-442.

[2] J. Fountain, Perfect semigroups, Proc. Edinburgh Math. Soc. 20 (1976), no. 2, 87-93.

[3] J. M. Howie, Fundamentals of Semigroup Theory, London Mathematical Society Monographs. London Math. Soc., London, 1995.

[4] J. Isbell, Perfect monoids, Semigroup Forum 2 (1971), no. 2, 95-118.

[5] R. Khosravi, M. Ershad, and M. Sedaghatjoo, Strongly flat and condition $(P)$ covers of acts over monoids, Comm. Algebra 38 (2010), no. 12, 4520-4530.

6] M. Kilp, Perfect monoids revisited, Semigroup Forum 53 (1996), no. 2, 225-229.

[7] _ On monoids over which all strongly flat cyclic right acts are projective, Semigroup Forum 52 (1996), no. 2, 241-245.

[8] M. Kilp, U. Knauer, and A. V. Mikhalev, Monoids, Acts and Categories, De Gruyter Expositions in Mathematics, vol. 29. de Gruyter, Berlin, 2000.

[9] M. Mahmoudi and J. Renshaw, On covers of cyclic acts over monoids, Semigroup Forum 77 (2008), no. 2, 325-338.

[10] H. S. Qiao and L. M. Wang, On flatness covers of cyclic acts over monoids, Glasg. Math. J. 54 (2012), no. 1, 163-167.

[11] J. Renshaw, Monoids for which condition $(P)$ acts are projective, Semigroup Forum 61 (2000), no. 1, 45-56.

[12] B. Stenström, Flatness and localization over monoids, Math. Nachr. 48 (1971), 315-334.

HUSHENG QIAO

Department of Mathematics

NoRThWEST NORMAL UNIVERSITY

Lanzhou 730070, Gansu, P. R. China

E-mail address: qiaohs@nwnu.edu.cn

Chongqing Wei

Department of Mathematics

Northwest Normal University

Lanzhou 730070, Gansu, P. R. China

E-mail address: wcq-7765363@126.com 